\title{
Effects of Monotonous Auditory Stimulation on the Human EEG
}

\author{
Marius Georgescu ${ }^{1}$, Daniel Georgescu ${ }^{1 *}$, Maria Iancau ${ }^{1}$, Anda Gadidov $^{2}$ \\ ${ }^{1}$ Department of Functional Disciplines, University of Medicine and Pharmacy of Craiova, Craiova, Romania; ${ }^{2}$ Department of \\ Mathematics and Statistics, Kennesaw State University, Kennesaw, USA. \\ Email: *d_g_home@yahoo.com
}

Received September $1^{\text {st }}, 2012$; revised October $3^{\text {rd }}, 2012$; accepted October $11^{\text {th }}, 2012$

\begin{abstract}
Monotony is the result of continuous predictive or repetitive stimulations and can be considered a functional state of the central nervous system, a state characterized by lowered cerebral activity. The present work investigates the effect of monotonous auditory input on cerebral electrical activity. This was done by means of recording the EEG under three different auditory stimuli (S1, S2, S3), on 11 volunteers (average age 23 years). Data were acquisitioned during 20 minutes of auditory stimulation, with a 5 minute period of silence before and after stimulation. The characteristic features of each obtained EEG pattern depends closely on the type of the musical composition. Thus, the highest domain of variance generated under stimulation by S3 (fragments of the Mozart's K448 Sonata) is for the frequency bands Alfa1, Beta2 and Theta, while in case of S2 (recording of a rainforest) it is for bands Delta, Beta1 and Alfa2, and in case of S1 (recording of a car driving over a rough road) is only slightly elevated for the total frequency spectrum. By comparing the period of auditory stimulation with the post-stimulation period, in case of S3 the fewest statistically significant mean values are noticeable (only for Delta and Theta indexes), in contrast to S1, which presents statistically significant differences between mean values for high frequency bands. In case of S2, statistically significant differences between mean values are similar to S3, with the addition of the Betal frequency band. In conclusion, each different auditory stimuli produces a characteristic pattern of changes in cortical micropotentials which could be associated with the induced psychoemotional state.
\end{abstract}

Keywords: Human EEG; Monotonous Auditory Stimulation; Music; Frequency Bands; Cortical Micropotentials

\section{Introduction}

Monotony was conceptualized as the result of constant, extremely predictive or repetitive stimulations. The environment in which an activity/task takes place may also influence performance as much as the monotony of the action itself [1]. It is a functional state of the central nervous system characterized by the decrease of the level of cerebral activation and is accompanied by sleepiness, a lower degree of attention and decreased vigilance [1,2].

Repetitive stimuli are largely recognized as being monotonous and associated with lack of attention and decreased performance [1]. Wertheim [3], however, suggests that monotony is determined by the predictability of environment stimuli and not by repetitivity. The complexity of a task/activity is also an important factor in monotony, its effects explained by two complementary theories: the theory of excitement and the theory of accommodation [4]. Thus, tasks with decreased requirements contribute to cognitive underload, lower attention,

${ }^{*}$ Corresponding author. lack of vigilance and monotony, while tasks with increased requirements and cognitive overload lead to tiredness [5].

Numerous studies concentrated on the effect of external stimulation on cortical EEG, especially regarding the response to visual stimulation [6,7]. The focus on using rhythmic visual and auditory stimulation as a means of inducing relaxation and hypnosis emerged during the latter half of the XXth century [8]. Variations in repetitive visual stimulation in neurophysiologic and psychophysiologic research are numerous, with repetitive visual stimulation patterns usually being presented in an on-off mode (e.g. flickering light) [9], or through inverted pattern changes (e.g. checkerboard pattern) [10]. Besides visual stimulation, auditory stimulation through repetitive acoustic stimuli, either naturally produced or artificially (e.g. music), either solely or associated with different tasks/activities can prove useful in accumulating new data regarding psychic sensorial and superior cognitive processes [11-13].

Music has proven a valuable instrument in the under- 
standing of human knowledge, human emotion and the cerebral mechanisms that underline them. Some researchers maintain that human musical abilities played a key phylogenetic role in the evolution of language and that musical behavior supported and promoted important evolutionary functions (e.g. communication, cooperation and social cohesion) [14]. From the oldest of times, music was used to stimulate emotions and treat a variety of illnesses: migraine, epilepsy, schizophrenia, dementia, autism, neurosis, anxiety, asthenia, stress, insomnia, depression, palpitations, arrhythmia, cardiac insufficiency, arterial hypertension [15-20]. Despite this, EEG investigations of cerebral activity under auditory stimulation in general and music in particular, are not abundant.

The present study aims at investigating cerebral electrical activity under the influence of three types of monotonous sounds, both recorded from nature and artificial, different in regards to frequencies, amplitudes, tonality and the induced psychoemotional state. The global influence of auditory stimulation on the EEG frequency bands was tracked and post stimulation effects on cerebral rhythms were studied.

\section{Experimental Procedures}

\subsection{Subjects}

The investigated group was made up of 11 males, all right handed, average age 23, students, and homogenous regarding professional and extraprofessional activity. The subjects perceived the entire band of frequencies between $45 \mathrm{~Hz}$ and $16,500 \mathrm{~Hz}$ at the same sound level $(40 \mathrm{~dB})$. After medical examination, subjects with neurological disturbances or a history of drug or ethanol abuse were excluded from the study. Starting 12 hours prior to the EEG recording, none of the following substances-alcohol, caffeine, tea, chocolate, B group vitamins, hormones, hypotensive drugs, sedatives, tranquilizers, sleeping pills were allowed. Permission for experiments with human subjects for scientific purposes was obtained from The Ethical Commission of the University of Craiova, Romania. Each subject was provided with detailed information about the aims of the ongoing study and gave his written consent to participate in it.

\subsection{Stimulation Procedure}

The auditory stimuli were sounds recorded from nature. Three sounds were utilized: S1 (recorded in an automobile moving on a rough surface), S2 (recorded in a rainforest) and S3 (recorded after Mozart's K448 Sonata). The EEG was recorded while the subject was undergoing continuous auditory stimulation, for the duration of 20 minutes, using a pair of "hook-on-ear" headphones connected to a netbook computer (an Acer Aspire one) running Windows Media Player 11 under Windows XP
Service Pack 3, powered by its own batteries to avoid parasitic currents. The intensity of the sound was measured with an NM102 Noise Meter.

\subsection{EEG Recording}

The acquisition of cortical biopotentials was made using an industrially produced electroencephalograph, NihonKohden EEG-9200. The electrodes were placed after the classic 10 - 20 system, bipolar acquisition [21], references being the 2 ears, and an extra ECG derivation (both hands and the right foot) with the main role of signal quality control. All recordings were made in identical experimental conditions: subjects with the same degree of physical and psychical tiredness, sitting still, eyes closed, no ambient sound and lighting, no disruptive ambient electrical fields.

The procedure was carried out as following 3 valid recordings for each subject, each made using a different sound of the three (S1, S2, S3), with the following steps: I: subjects close their eyes at the operator's command; II after 5 minutes of silence (L1 period) the operator starts the auditory stimulation; III: after 20 minutes of stimulation (S period) the operator stops the auditory stimulation; IV: after 5 minutes of silence (L2 period) the operator stops the recording. To avoid inducing a rhythm modulation subjects were not instructed to any particular mental activity and were given complete freedom.

\subsection{Data Analysis}

Cerebral rhythms were highlighted through spectral analysis obtained on the basis of the Fourier Transform in its FFT variant (Fast Fourier Transform).

The analysis of the influence of auditory stimulation by S1, S2, S3 on cortical micropotentials included the global comparison of the mean frequency EEG bands for the entire stimulation period (20 minutes), determined on the auditory projection area (the area covered by the P3, $\mathrm{P} 4, \mathrm{O} 1$ and $\mathrm{O} 2$ electrodes). In this manner three sets of values were established for the total spectrum of frequencies, and for the individual frequency bands Delta, Theta, Alfa1, Alfa2, Beta1 and Beta2 respectively. To compare mean frequency bands for the 20 minutes of stimulation with each of the three types of auditory stimuli, the ANOVA one-way variance analysis test was utilized, both for the total frequency spectrum and the individual bands Delta, Theta, Alfa1, Alfa2, Beta1 and Beta2. The graphic presentation of values was made in the Boxplot variant.

To determine the measure in which long duration monotonous auditory stimulation manifests an effect on the EEG (and then ceases this effect), a comparison was made through the application of the Student test, between the mean EEG frequencies during stimulation (S) and 
immediately after stimulation ceases (L2).

\section{Results}

\subsection{Global Analysis of the EEG Frequency Bands during Auditory Stimulation}

The first comparison of the mean values through the ANOVA test was made for the Total index, which represents the mean for the 20 minutes of stimulation of the total frequency spectrum.

The first thing that must be noticed following analysis is the statistically significant difference $(p=0.042)$ between the obtained mean values (Figure 1(a)). For each of the 3 situations (S1, S2, and S3) the variation domain can be observed, the 3 - 4 quartiles, the median and the mean value. The individual bands of frequency were analyzed in order to determine the measure of their consistency with the Total index. Thus, for the Alfa1 frequency band, the Alfa1 index has mean values that are statistically highly significant $(p=0.001)$ for the periods in which stimulation was made by each of the three stimuli. Figure 1(b) presents the variation of the Alfal frequency band under stimulation with the 3 sounds. The Alfa2 component did not offer values that are statistically significant for all three stimulations ( $p$ is over 0.080 ) (Figure 1(c)).

The ANOVA test offers statistically significant differences between the mean values of the Betal indexes under stimulation by $\mathrm{S} 1, \mathrm{~S} 2$ and $\mathrm{S} 3(\mathrm{p}=0.027)$ (Figure 1(d)), while for the upper part of the beta band, Beta2, there are no statistically significant differences between means $(p=0.341)$. Figure 1(e) highlights the variation of the Beta2 band under stimulation by the 3 sounds. Applying the ANOVA test to the Delta and Theta indexes it can be observed that for both indexes the means of the three situations vary with high statistical significance between themselves ( $p$ is 0.000 and 0.003 respectively) (Figures 1(f) and (g)). A comparison of all variation domains and mean values is represented in Figure 1(h).

\subsection{Comparative Analysis of the EEG Frequency Bands between the Auditory Stimulation Period (S) and Post-Stimulation Period (L2)}

In order to compare the mean values for the EEG frequency bands (noted as Total: Tot, Alfa1: A1, Alfa2: A2, Beta1: B1, Beta2: B2, Delta: D, Theta: T) between S (under all 3 sounds) and L2 the Student test was used. The obtained values are listed in Table 1, of which statistically significant values were written on a grey background. Comparative graphic representation between the 2 situations, S and L2, is made in Figure 2, which shows that mean values for the two analyzed periods differ visibly for almost all parameters, with the exception of D band for S1 and B2 band for S2.

\section{Discussion}

The three sounds utilized were considered representative to achieving a monotonous unpleasant auditory stimulation in case of S1, a monotonous soothing stimulation in case of S2, and a pleasant but tensing stimulation in case of S3, respectively. To track the influence of monotonous auditory stimulation under all 3 types of sounds on cortical micropotentials initial comparisons were made between the means of the frequency bands (for total spectrum and each individual EEG band, respectively) for the entire stimulation period through the one-way ANOVA test. Thus, highly significant data was found for the Alfa1, Delta and Theta bands, while no significant differences were found for bands Alfa2 and Beta2. As for the total spectrum, although variation domains are relatively similar, with a slight superiority for S1, mean values differ significantly, the highest value being for the artificial sound (S3) and not the one recorded from nature (S1) (Figures 1(a) and (h)).

Analysis shows (Figure 1) that for each frequency band the lowest mean value is in case of S1, middle value in case of S2, and highest value in case of S3, for the majority of frequency bands with the exception of Delta and Beta2. The highest variation domain is generated in case of stimulation by S3 for bands Alfa1, Beta2 and Theta. Some authors relate Theta [22] and Alfa1 activity [23] to emotional tension or anxiety. Literature data on the influence of music on the electrical activity of the human brain is conflicting. Some authors $[24,25]$ have reported an increase in power for the Alpha rhythm while listening to music, while others [26] have observed an increase in power of Theta activity against the background of a decrease in the total power of the Alpha rhythm.

Exposure to other classical music pieces (fragments from Mozart's Concertos for Piano and Orchestra nos. 20 and 21) of comparable intensity level resulted in virtually no widely generalized changes of the EEG power in the Delta and Alfa bands, while the increase in power of the high-frequency EEG bands (Alfa2, Beta1, Beta2, Gamma) embraced almost the entirety of the cortex [27]. What conflicting results exist between our analysis and [27] could be partially explained by the difference in stimulation length and partially by the difference in the parameters of the stimuli: a solo instrument piece versus multiple instruments in orchestra.

The observed discrepancies in various literature data are possibly related to the fact that most works don't make a specific distinction of music into styles, and often the intensity and duration of the musical piece was not taken into account, all of which makes the comparison with literature data hard to make. 


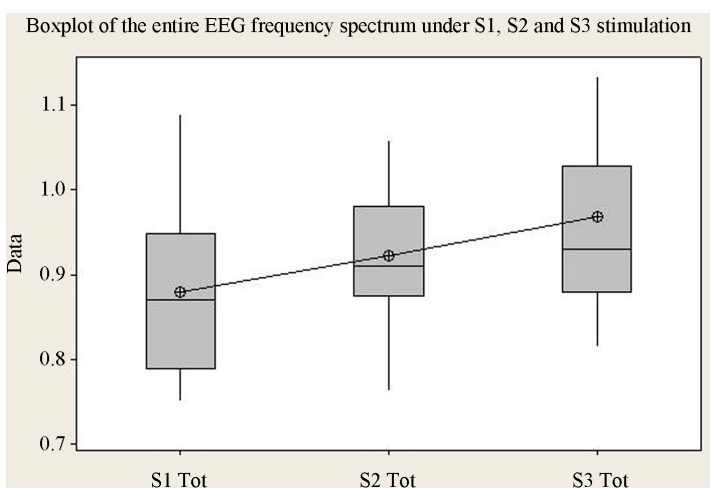

(a)

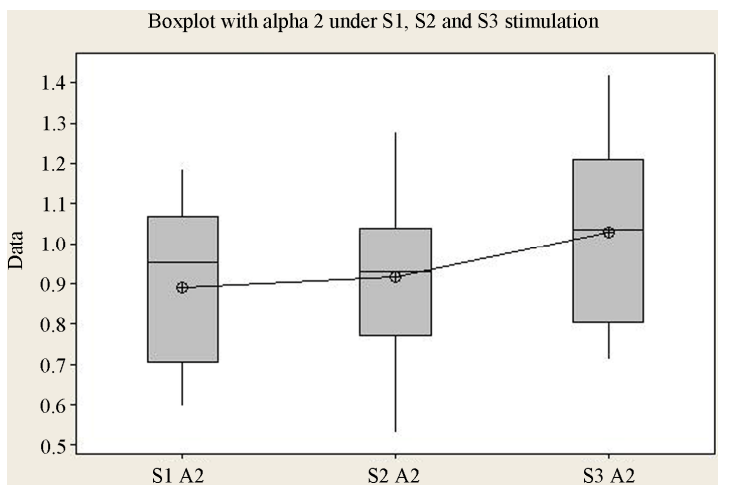

(c)

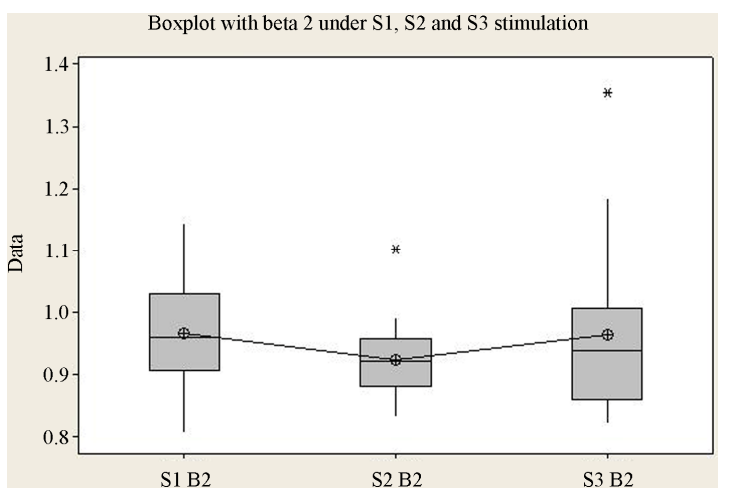

(e)

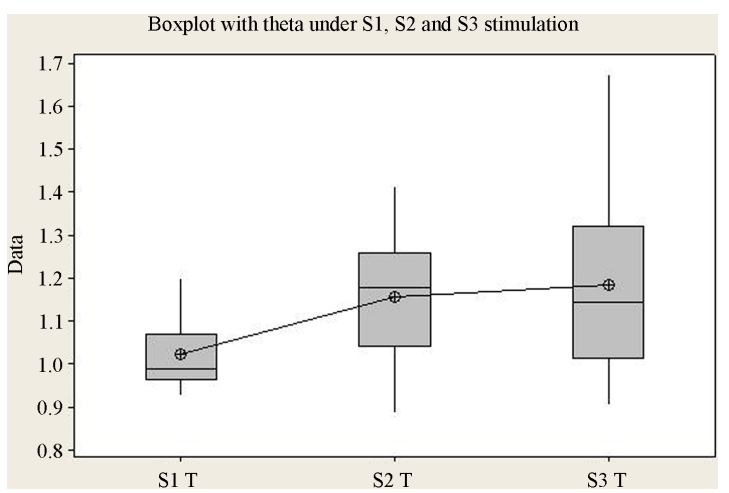

(g)

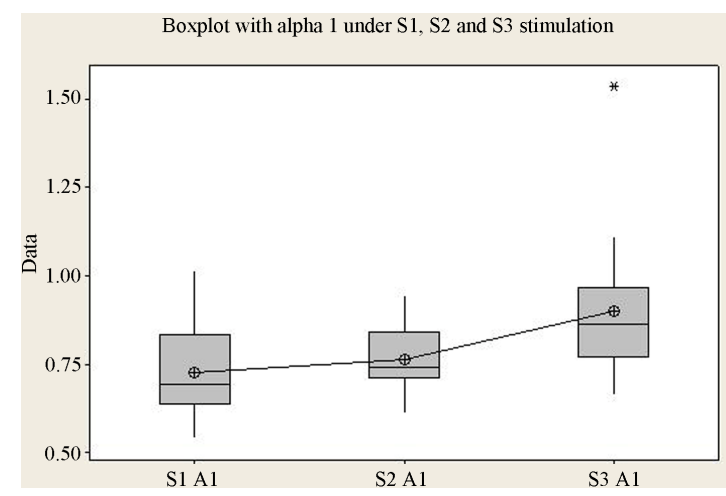

(b)

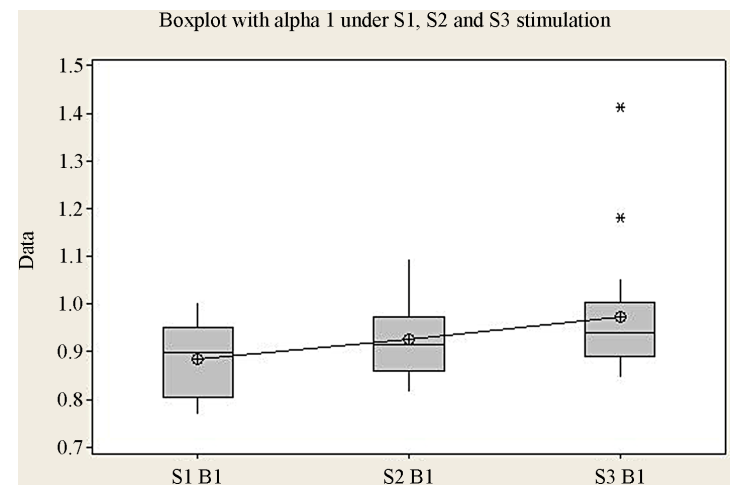

(d)

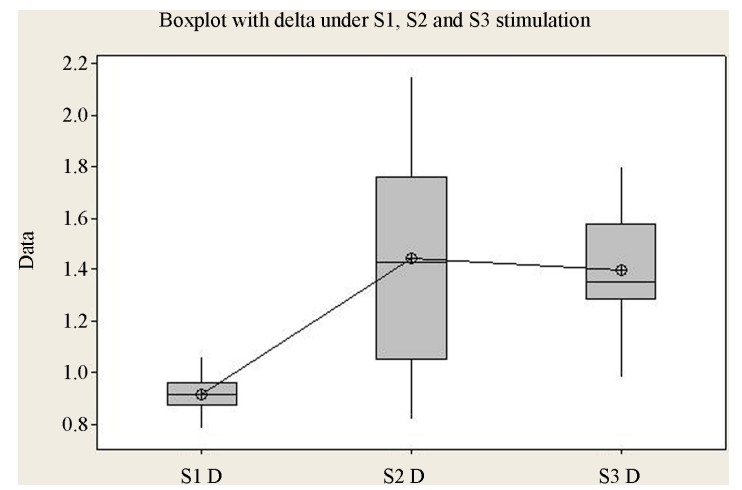

(f)

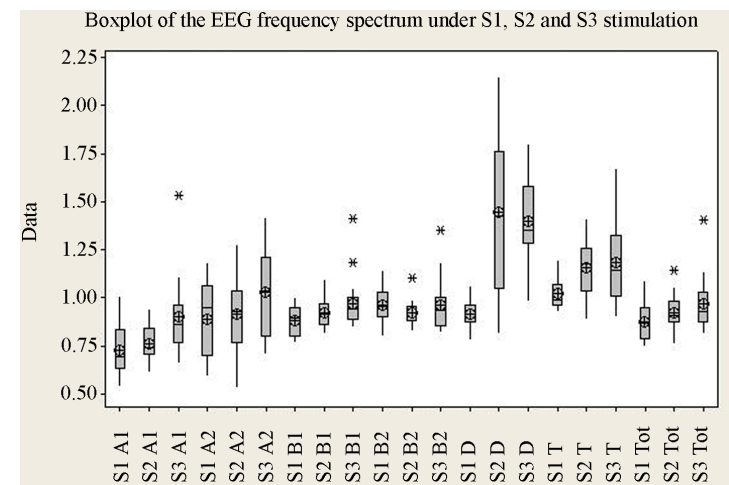

(h)

Figure 1. The variation of EEG frequency bands under stimulation by S1, S2, S3. (a) Total spectrum; (b) Alfa1; (c) Alfa2; (d) Beta1; (e) Beta2; (f) Delta; (g) Theta; (h) Overview image of the variation of all EEG frequency bands. *Values which were considered outside the variation domain by the statistic test. 
Table 1. Comparative synthetic data between the stimulation period (S) under S1, S2, S3, and post-stimulation period (L2).

\begin{tabular}{|c|c|c|c|c|c|}
\hline $\begin{array}{l}\text { EEG } \\
\text { Band }\end{array}$ & Stimulation & $\underset{\text { t-test S-L2 }}{p}$ & $\begin{array}{l}\text { EEG } \\
\text { Band }\end{array}$ & Stimulation & $\underset{\text { t-test } S-L 2}{p}$ \\
\hline \multirow{3}{*}{ A1 } & S1 & 0.017454 & & S1 & 0.03563 \\
\hline & $\mathrm{S} 2$ & 0.226838 & B2 & $\mathrm{S} 2$ & 0.43469 \\
\hline & S3 & 0.123295 & & S3 & 0.288255 \\
\hline \multirow{3}{*}{ A2 } & S1 & 0.005969 & & S1 & 0.419526 \\
\hline & $\mathrm{S} 2$ & 0.169172 & D & $\mathrm{S} 2$ & 0.002062 \\
\hline & S3 & 0.120636 & & S3 & 0.000593 \\
\hline \multirow{3}{*}{ B1 } & S1 & 0.004041 & & S1 & 0.283054 \\
\hline & S2 & 0.041114 & $\mathrm{~T}$ & $\mathrm{~S} 2$ & 0.003897 \\
\hline & $\mathrm{S} 3$ & 0.303689 & & S3 & 0.000894 \\
\hline \multicolumn{2}{|c|}{ EEG Band } & \multicolumn{2}{|c|}{ Stimulation } & \multicolumn{2}{|c|}{$\underset{\text { t-test S-L2 }}{p}$} \\
\hline \multirow{3}{*}{\multicolumn{2}{|c|}{ Tot }} & \multicolumn{2}{|c|}{ S1 } & \multicolumn{2}{|c|}{0.002674} \\
\hline & & \multicolumn{2}{|c|}{$\mathrm{S} 2$} & \multicolumn{2}{|c|}{0.037685} \\
\hline & & \multicolumn{2}{|c|}{ S3 } & \multicolumn{2}{|c|}{0.295132} \\
\hline
\end{tabular}

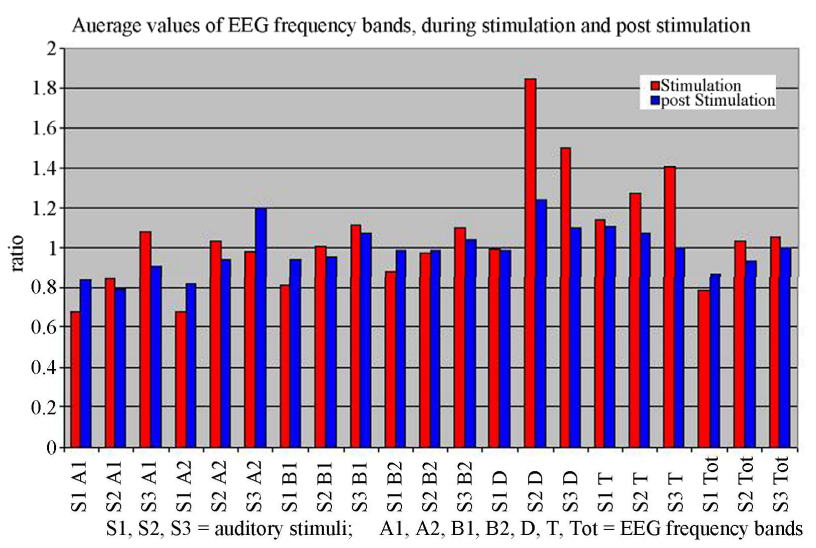

Figure 2. Comparative representation of the mean values of EEG frequency bands during stimulation (S) and post stimulation (L2).

The second sound, S2, representative for soothing stimulation, generates the highest variation domain only in cases of the Delta, Beta1 and Alfa2 bands. Similarly, literature data show an increase in the EEG power of the Delta band while listening to subjectively pleasant music [28]. The lowest variation domains are for S1 in Delta and Theta bands, for S2 in Alfa1 and Beta2 bands, and for S3, the lowest variation domain only appears in case of Beta1. It is surprising to note that for Alfa1 the lowest variation domain belongs to $\mathrm{S} 2$ stimulation, which is a soothing sound, recorded from nature, that we would be tempted to credit with the highest potential to generate alpha waves. Unlike Alfa1 band, the variation of the Alfa2 frequency band presents the largest domain for S2, which we were expecting since the sound was described as subjectively profoundly soothing.

To compare the stimulation period with the post-stimulation period the significant differences recorded between mean values corresponding to EEG frequency bands (Tot, $\mathrm{A} 1, \mathrm{~A} 2, \mathrm{~B} 1, \mathrm{~B} 2, \mathrm{D}$ and $\mathrm{T}$ ) for the two situations were tracked.
By analyzing Table 1 it can be observed that, for the EEG total spectrum, S1 and S2 stimulation offers mean values which differ statistically significant, while S3 does not provide statistical meaning. In fact, S3 stimulation offers the least statistically significant means (only in the case of $\mathrm{D}$ and $\mathrm{T}$ indexes).

The diversity of Mozart's compositions shows, breaks in rhythm and tonality ensuring the lack of monotony and thus no delaying effect on EEG response. S1, chosen to analyze the effects of an unpleasant stimulus, offers differing statistically significant results in all but two cases (D and T). This shows a completely different cerebral response in comparison to the period without stimulation and that it does not induce a latency effect that would delay response. S2, chosen as a soothing, pleasant stimulus, does not offer results nearly as definite. Even though for the general situation in which the total EEG spectrum is compared there are statistically significant differences, half of the specific indicators (A1, A2, and B2) responses are not statistically significant.

To summarize, each different auditory stimuli produces a characteristic pattern of changes in cortical micropotentials. It would appear that each sound exerts a prominent influence on certain EEG frequency bands: S3 influences the low-frequency bands, $\mathrm{S} 1$ the high-frequency ones, while S2 exerts a moderate influence on both lowand high-frequency bands with a slight emphasis on the former.

As preliminary data, the present investigation can broaden the area of knowledge regarding the effects of monotonous auditory stimulation on cerebral activity as well as help in accumulating new data regarding psychic sensorial and superior cognitive processes.

\section{Acknowledgements}

This work was partially supported by the strategic grant POSDRU/88/1.5/S/52826, Project ID52826 (2009), co- 
financed by the European Social Fund-Investing in People, within the Sectorial Operational Programme Human Resources Development 2007-2013.

\section{REFERENCES}

[1] P. Thiffault and J. Bergeron, "Monotony of Road Environment and Driver Fatigue: A Simulator Study," Accident Analysis and Prevention, Vol. 35, No. 3, 2003, pp. 381-391. doi:10.1016/S0001-4575(02)00014-3

[2] S. K. L. Lal and A. Craig, "A Critical Review of the Psychophysiology of Driver Fatigue," Biological Psychology, Vol. 55, No. 3, 2001, pp. 173-194. doi:10.1016/S0301-0511(00)00085-5

[3] A. H. Wertheim, "Highway Hypnosis: A theoretical Analysis," In: A. G. Gale, I. D. Brown, C. M. Haslegrave, I. Moorhead and S. Taylor, Eds., Vision in Vehicles-III Amsterdam: Elsevier Science Publishers B.V, 1991, pp. 467472.

[4] A. S. Smit, P. A. T. M Eling and A. M. L. Coenen, "Mental Effort Causes Vigilance Decrease Due to Resource Depletion," Acta Psychologica, Vol. 115, No. 1, 2004, pp. 35-42. doi:10.1016/j.actpsy.2003.11.001

[5] G. J. Wilde and J. F. Stinson, "The Monitoring of Vigilane in Locomotive Engineers," Accident Analysis and Prevention, Vol. 15, No. 2, 1983, pp.87-93. doi:10.1016/0001-4575(83)90065-9

[6] E. Tekin, M. Engin, T. Dalbasti and E. Z. Engin, "The Evaluation of EEG Response to Photic Stimulation in Normal and Diseased Subjects," Computers in Biology and Medicine, Vol. 39, No. 1, 2009, pp. 53-60. doi:10.1016/j.compbiomed.2008.11.001

[7] N. Martini, D. Menicucci, L. Sebastiani, R. Bedini, A. Pingitore, N. Vanello, M. Milanesi, L. Landini and A. Gemignani, "The Dynamics of EEG Gamma Responses to Unpleasant Visual Stimuli: From Local Activity to Functional Connectivity," Neuroimage, Vol. 60, No. 2, 2012, pp. 922-932. doi:10.1016/j.neuroimage.2012.01.060

[8] M. Teplan, A. Krakovská and S. Stolc, "Direct Effects of Audio-Visual Stimulation on EEG," Computer Methods and Programs in Biomedicine, Vol. 102, No. 1, 2011, pp. 17-24. doi:10.1016/i.cmpb.2010.11.013

[9] Y. Soeta, S. Uetani and Y. Ando, "Propagation of Repetitive Alpha Waves Over the Scalp in Relation to Subjective Preferences for a Flickering Light," International Journal of Psychophysiology, Vol. 46, No. 1, 2002, pp. 41-52. doi:10.1016/S0167-8760(02)00063-6

[10] X. Bai, Z. Liu, N. Zhang, W. Chen and B. He, "ThreeDimensional Source Imaging from Simultaneously Recorded ERP and BOLD-FMRI," IEEE Transactions on Neural Systems and Rehabilitation Engineering, Vol. 17, No. 2, 2009, pp. 101-106. doi:10.1109/TNSRE.2009.2015196

[11] M. J. Corral and C. Escera, "Effects of Sound Location on Visual Task Performance and Electrophysiological Measures of Distraction," Neuroreport, Vol. 19, No. 15, 2008, pp. $1535-1539$.

\section{doi:10.1097/WNR.0b013e3283110416}

[12] N. Jaušovec, K. Jaušovec and I. Gerlic, "The Influence of Mozart's Music on Brain Activity in the Process of Learning," Clinical Neurophysiology, Vol. 117, No. 12, 2006, pp. 2703-2714. doi:10.1016/j.clinph.2006.08.010

[13] N. Jaušovec and K. Jaušovec, "Differences in Induced Gamma and Upper Alpha Oscillations in the Human Brain Related to Verbal/Performance and Emotional Intelligence," International Journal of Psychophysiology, Vol. 56, No. 3, 2005, pp. 223-235. doi:10.1016/j.ijpsycho.2004.12.005

[14] S. Koelsch, K. Offermanns and P. Franzke, "Music in the Treatment of Affective Disorders: an Exploratory Investigation of a New Method for Music-Therapeutic Research," Music Perception, Vol. 27, No. 4, 2010, pp. 307316. doi:10.1525/mp.2010.27.4.307

[15] J. R. Hughes, “The Mozart Effect: Additional Data," Epilepsy \& Behavior, Vol. 3, No. 2, 2002, pp. 182-184. doi:10.1006/ebeh.2002.0329

[16] B. Rimland and S. M. Edelson, "A Pilot Study of Auditory Integration Training in Autism," Journal of Autism and Developmental Disorders, Vol. 25, No. 1, 1995, pp. 61-70. doi:10.1007/BF02178168

[17] S. Schneider, P. W. Schönle, E. Altenmüller and T. F. Münte, "Using Musical Instruments to Improve Motor Skill Recovery Following a Stroke," Journal of Neurology, Vol. 254, No. 10, 2007, pp. 1339-1346. doi:10.1007/s00415-006-0523-2

[18] S. M. Peng, M. Koo and J.C. Kuo, "Effect of Group Music Activity as an Adjunctive Therapy on Psychotic Symptoms in Patients with Acute Schizophrenia," Archives of Psychiatric Nursing, Vol. 24, No. 6, 2010, pp. 429-434. doi:10.1016/j.apnu.2010.04.001

[19] K. Sherratt, A. Thornton and C. Hatton, "Music Interventions for People with Dementia: A Review of the Literature," Aging \& Mental Health, Vol. 8, No. 1, 2004, pp. 312. doi:10.1080/13607860310001613275

[20] E. Bodner, J. Iancu, A. Gilboa, A. Sarel, A. Mazor and D. Amir, "Finding Words for Emotions: the Reactions of Patients with Major Depressive Disorder Towards Various Musical Excerpts," Arts in Psychotherapy, Vol. 34, No. 2, 2007, pp. 142-150. doi:10.1016/j.aip.2006.12.002

[21] E. Niedermeyer and F. L. da Silva, "Electroencephalography: Basic Principles, Clinical Applications, and Related Fields," Lippincott Williams \& Wilkins, Philadelphia, 2011.

[22] N. E. Sviderskaya, V. N. Prudnikov and A. G. Antonov, "EEG Features of Signs of Human Anxiety," Fiziologiia Cheloveka, Vol. 16, No. 3, 1990, p. 12.

[23] N. V. Dubrovinskaya, "Neurophysiological Mechanisms of Attention: An Ontogenetic Study," Nauka, 1985, p. 144.

[24] T. Ikeda, "Concentration-Effect and Underestimation of Time by Acoustic Stimuli," Shinrigaku Kenkyu, Vol. 63, No. 3, 1992, pp. 157-162. doi:10.4992/jipsy.63.157

[25] A. V. Sulimov, Y. V. Lyubimova, R. A. Pavlygina and V. I. Davydov, "Spectral Analysis of Human EEG during Listening to Music," Zhurnal Vysshě Nervnoü Deyatel- 
nosti Imeni I P Pavlova, Vol. 50, No. 1, 2000, pp. 62-67.

[26] Q. Yuan, X. H. Liu, D. C. Li, H. L. Wang and Y. S. Liu, "Effects of Noise and Music on EEG Power Spectrum," Space Medicine \& Medical Engineering, Vol. 19, No. 6, 2000, pp. 401-404.

[27] R. A. Pavlygina, D. S. Sakharov and V. I. Davydov, "Spectral Analysis of the Human EEG during Listening to Musical Compositions," Human Physiology, Vol. 30,
No. 1, 2004, pp. 54-60. doi:10.1023/B:HUMP.0000013765.64276.e6

[28] M. Kabuto, T. Kageyama and H. Nitta, "EEG Power Spectrum Changes during to Listening to Pleasant Music and Their Relation to Relaxation Effects," Nippon Eiseigaku Zasshi (Japanese Journal of Hygiene), Vol. 48, No. 4, 1993, pp. 807-818. doi:10.1265/jih.48.807 